\title{
Expression profiles of metastatic brain tumor from lung adenocarcinomas on cDNA microarray
}

\author{
TAKEFUMI KIKUCHI $^{1,4}$, YATARO DAIGO ${ }^{1}$, NOBUHISA ISHIKAWA ${ }^{1}$, TOYOMASA KATAGIRI ${ }^{1}$, \\ TATSUHIKO TSUNODA ${ }^{2}$, SEIICHI YOSHIDA ${ }^{3}$ and YUSUKE NAKAMURA ${ }^{1}$ \\ ${ }^{1}$ Laboratory of Molecular Medicine, Human Genome Center, Institute of Medical Science, \\ The University of Tokyo, Tokyo 108-8639; ${ }^{2}$ Laboratory for Medical Informatics, SNP Research Center, \\ Riken (Institute of Physical and Chemical Research), Kanagawa 230-0045; ${ }^{3}$ Department of Neurosurgery, \\ Niigata Cancer Center Hospital, Niigata 951-8566; ${ }^{4}$ First Department of Internal Medicine, \\ Sapporo Medical University, Hokkaido 060-8543, Japan
}

Received September 30, 2005; Accepted November 7, 2005

\begin{abstract}
Distant metastasis is one of the crucial parameters determining the type of treatment and prognosis of patients. Previous studies discovered important factors involved in multiple steps of metastasis, the precise mechanisms of metastasis still remain to be clarified. To identify genes associated with this complicated biological feature of cancer, we analyzed expression profiles of 16 metastatic brain tumors derived from primary lung adenocarcinoma (ADC) using cDNA microarray representing 23,040 genes. We applied bioinformatic algorithm to compare the expression data of these 16 brain metastatic loci with those of 37 primary NSCLCs including 22 ADCs, and found that metastatic tumor cells has very different characteristics of gene expression patterns from primary ones. Two hundred and forty-four genes that showed significantly different expression levels between the two groups included plasma membrane bounding proteins, cellular antigens, and cytoskeletal proteins that might play important roles in altering cell-cell communication, attachment, and cell motility, and enhance the metastatic ability of cancer cells. Our results provide valuable information for development of predictive markers as well as novel therapeutic target molecules for metastatic brain tumor of ADC of the lung.
\end{abstract}

\section{Introduction}

Distant metastasis is one of crucial steps in tumor development and progression causing difficulty in treatment and resulting in patient death $(1,2)$. Since there is no curative therapy for

Correspondence to: Dr Yusuke Nakamura, Laboratory of Molecular Medicine, Human Genome Center, Institute of Medical Science, The University of Tokyo, 4-6-1 Shirokanedai, Minato-ku, Tokyo 108-8639, Japan

E-mail: yusuke@ims.u-tokyo.ac.jp

Key words: gene expression profile, therapeutic target, prognostic factor, metastatic brain tumor, lung cancer metastatic tumors at present, novel strategies for diagnosis at an earlier stage and better therapies to improve patient survival with metastatic cancer are urgently required. Distant metastasis is considered to occur as multiple-step events which include invasion from a primary site, penetration into blood or lymphatic flow, circulation to reach distant organs, extravasation into a target organ, and re-growth $(3,4)$. To clarify this complicated but clinically important metastatic process, many investigators have attempted to identify the molecules of importance in these steps, but precise mechanisms of metastasis still remain largely unknown (5-7).

Because microarray technology allows us to analyze expression level of thousands of genes simultaneously, we previously applied this technology to characterize nonsmall cell lung carcinomas (NSCLCs), which include adenocarcinoma (ADC) and squamous cell carcinoma (SCC), and indicated that biological characteristics of each lung cancer like the risk of lymph node metastasis could be predictable by the expression profile of the tumor cells (8). In addition to the lymph node metastasis, lung cancer is known to show higher rate of brain metastasis; nearly $30 \%$ of metastatic brain tumors are derived from primary lung cancer. Lung ADC is generally characterized by the early development of brain metastasis, that is observed even in some cases whose primary tumor is symptom-free (9). The incidence of brain metastasis, based on autopsy findings, is as high as $50 \%$ in patients with ADC $(10,11)$. Few patients who develop brain metastasis from any primary tumor survive $>2$ years from diagnosis of metastasis, and their median survival time is only 2 months (12). Hence, prediction method of brain metastasis and application of such information to prevent the metastasis are very important for improvement of prognosis and quality of life for patients with lung cancer. At present, it is not possible to precisely predict which patients are likely to develop brain metastasis from lung cancers, although a number of biomarkers have been reported to be such predictive factors (13-17).

In this study, we focused on expression profile analysis of metastatic brain tumors derived from primary lung ADC and describe the identification of genes differently expressed between primary and metastatic sites. These data should be 
useful for better understanding of the molecular mechanism of brain metastasis and for development of new strategies for treatment of metastatic tumors.

\section{Materials and methods}

Patients and tissue samples. Metastatic brain tumors derived from lung ADC and their clinical information were obtained with informed consent from 16 patients who underwent surgery at the Niigata Cancer Center Hospital (Niigata, Japan). Each tumor was diagnosed according to histopathological subtype and grade. $\mathrm{pT}$ and $\mathrm{pN}$ factors of primary cancer in the lung was determined on the basis of UICC TNM classification as follows: 8 cases to be T1N0, 4 cases to T2N0, and 4 cases to T3N1-3. All samples were immediately frozen and embedded in TissueTek OCT medium (Sakura, Tokyo, Japan) and stored at $-80^{\circ} \mathrm{C}$. This study and the use of all clinical materials mentioned were approved by individual institutional ethics committees.

Laser-microbeam microdissection, extraction of RNA, and T7-based RNA amplification. Cancer cells were selectively collected from the preserved samples using laser-microbeam microdissection (LMM) method (18). Extraction of total RNA and T7-based amplification were performed as described previously $(8,19)$. As a control probe, normal human lung poly(A) RNA (BD Biosciences Clontech, Palo Alto, CA, USA) was amplified using the same amplification condition; $2.5-\mu \mathrm{g}$ aliquots of amplified RNAs (aRNAs) from each cancerous tissue and from the control were reverse transcribed in the presence of Cy5-dCTP and Cy3-dCTP, respectively.

cDNA microarray. cDNAs spotted on the glass slides were prepared by RT-PCR using a unique primer set that corresponded to cDNA sequence of each gene as described previously $(8,20)$. The PCR products were spotted on type VII glass-slides (Amersham Biosciences, Uppsala, Sweden) with a Microarray Spotter Generation III (Amersham Biosciences); 4,608 genes were spotted in duplicate on a single slide. We prepared five different sets of slides (a total of 23,040 spots), each of which contained the same 52 housekeeping genes as well as two non-human negative control genes. Hybridization, washing and detection of signals were carried out as described previously $(8,18-21)$. The fluorescence intensities of Cy5 (tumor) and Cy3 (control) for each target spot were adjusted so that the mean $\mathrm{Cy} 3 / \mathrm{Cy} 5$ ratio of the 52 housekeeping genes was equal to one. Because the data derived from low signal intensities are less reliable, we determined a cut-off value for signal intensities on each slide and excluded genes from further analysis when both $\mathrm{Cy} 3$ and $\mathrm{Cy} 5$ dyes gave signal intensities lower than the cut-off.

Cluster analysis of metastatic brain tumors from lung ADC and primary NSCLCs. We applied a hierarchical clustering method $(8,20,21)$ to both genes and 53 lung tumors (16 metastatic lung ADC and an independent set of 37 primary sites of NSCLC analyzed previously) (8). To obtain reproducible clusters for classification of these 53 samples, we selected 4,423 genes for which valid data were obtained in $90 \%$ of the experiments, and whose expression ratios varied by standard deviations of $>0.8$. The analysis was performed using webavailable software ('Cluster' and 'TreeView') written by M. Eisen (http://genome-www5.stanford.edu/MicroArray/SMD/ restech.html). Prior to applying the clustering algorithm, we log-transformed the fluorescence ratio for each spot and then median-centered the data for each sample to remove experimental bias.

Identification of genes differently expressed between primary lung $A D C$ and brain metastasis of lung $A D C$. We applied a random permutation test to identify genes that were expressed differently between a group of 16 brain metastatic sites of lung ADC and an independent set of 22 primary lung ADC analyzed previously (8). Mean $(\mu)$ and standard $(\sigma)$ deviations were calculated from the log-transformed relative expression ratios of each gene in primary $(\mathrm{p})$ and metastatic $(\mathrm{m})$ tumors. To reduce the dimensionality of the number of potent genes that could discriminate between the two classes, we extracted only genes that fulfilled two criteria: a) signal intensities higher than the cut-off level in at least $50 \%$ of the cases; b) $\mid$ Medm-Medp $\mid \geq 2$, where Med indicates the median derived from log-transformed relative expression ratios in primary $\operatorname{ADC}(\mathrm{p})$ or metastatic brain tumor from $\operatorname{ADC}(\mathrm{m})$. Then we carried out permutation tests to estimate the ability of individual genes to distinguish between the two groups; samples were randomly permutated between the two classes 10,000 times.

\section{Results}

Clustering analysis of metastatic lung $A D C$ and primary NSCLC. To exclude contamination of normal brain cells surrounding metastatic tumor cells, we employed the LMM system and collected almost pure population of tumor cells. To analyze similarities among samples and among genes, we firstly applied two-dimensional hierarchical clustering algorithm using the expression profile data obtained from 16 metastatic brain tumors from ADC and 37 primary NSCLCs. Expression data in Fig. 1A consists of 4,423 genes that passed through the cut-off filter described in Materials and methods. The dendrogram shown at the top of the figure represents similarities between samples and shorter branch indicates higher similarity. In the tumor axis, three major groups corresponding to a group of primary ADC, that of primary SCC including one adenosquamous carcinoma (ASC) and that of metastatic brain tumors from lung ADC were clearly separated by the information of expression profiles. In the magnified view of this cluster analysis (Fig. 1B, gene cluster-1, -2, and -3), identical genes spotted on different positions on glass slides showed very high similarities in the gene cluster, representing reproducibility and reliability of our experimental procedures. In addition, we identified genes whose expression levels were relatively decreased (Fig. 1A and B, gene cluster-4) or increased (Fig. 1A and B, gene cluster-5) in metastatic brain tumors. The genes that revealed decreased expression levels in brain metastatic legions comparing to the primary tumors shown as gene cluster-4 in Fig. 1B included SLC9A1, TNFRSF1A, C14ORF1, and $M M P 24$ (MT5-MMP), which were cancer-related genes or whose expression was reported to be down-regulated in several tumor types (22-25). SLC9A1, a Na/H exchanger, was 
A
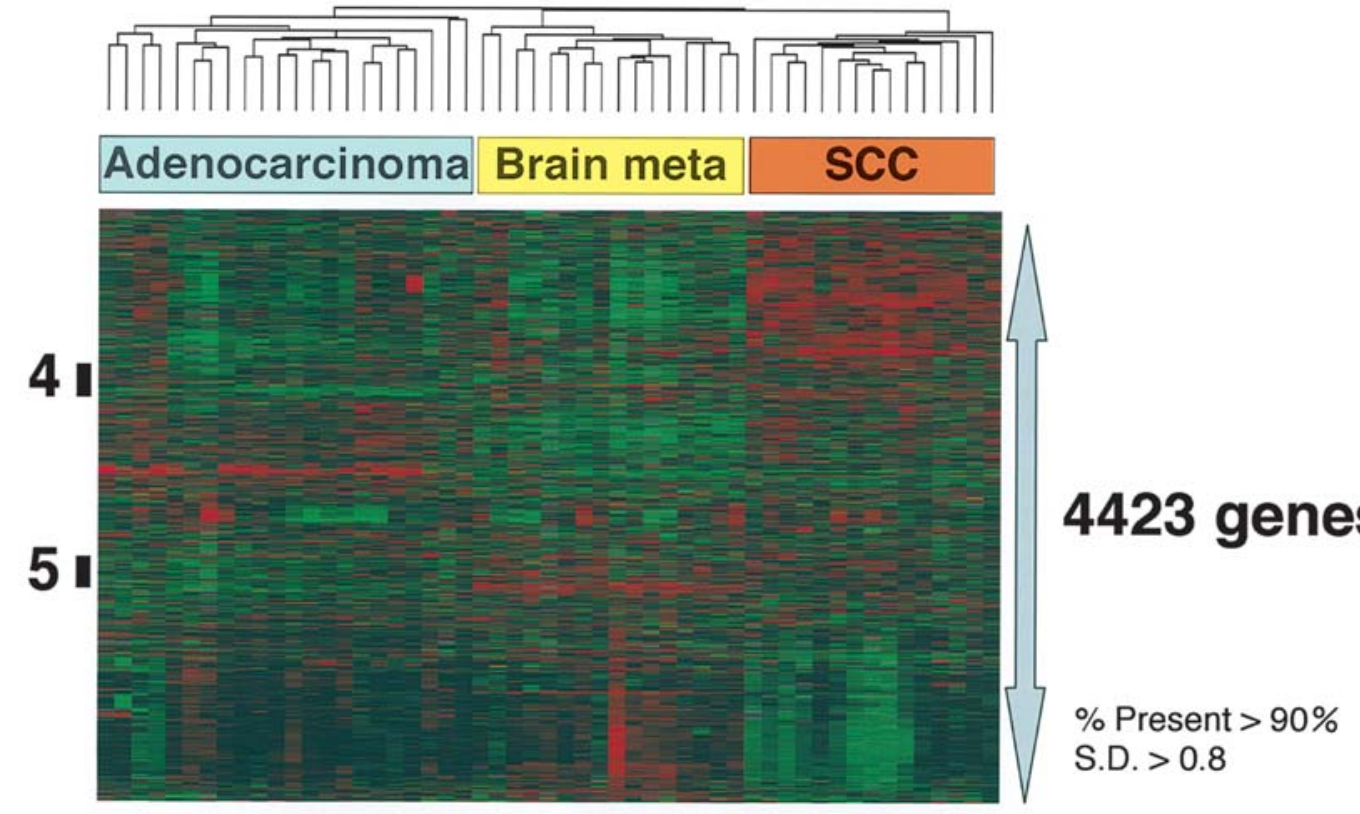

B

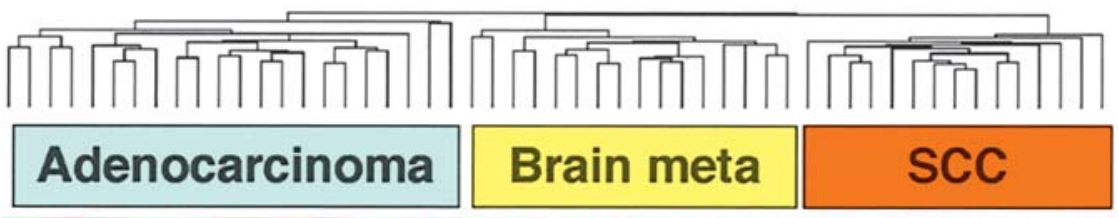

1

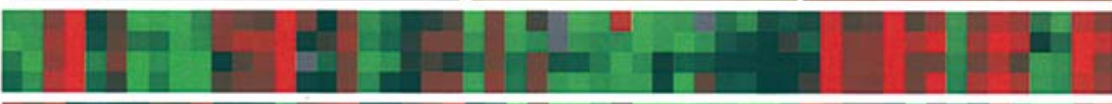

2

3

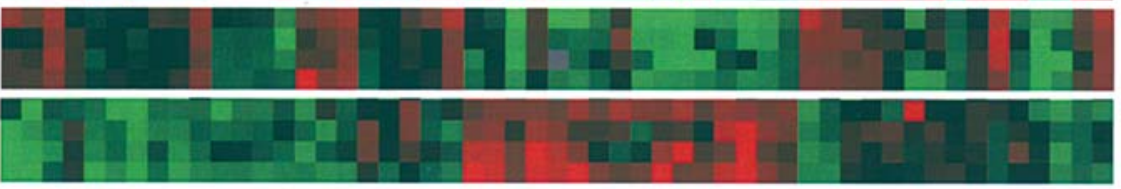

HIST2H2BE
HIST2H2BE HIST2H2BE HIST2H2B HMGCL HMGCL
HMGCL HMGCL
HMGCL

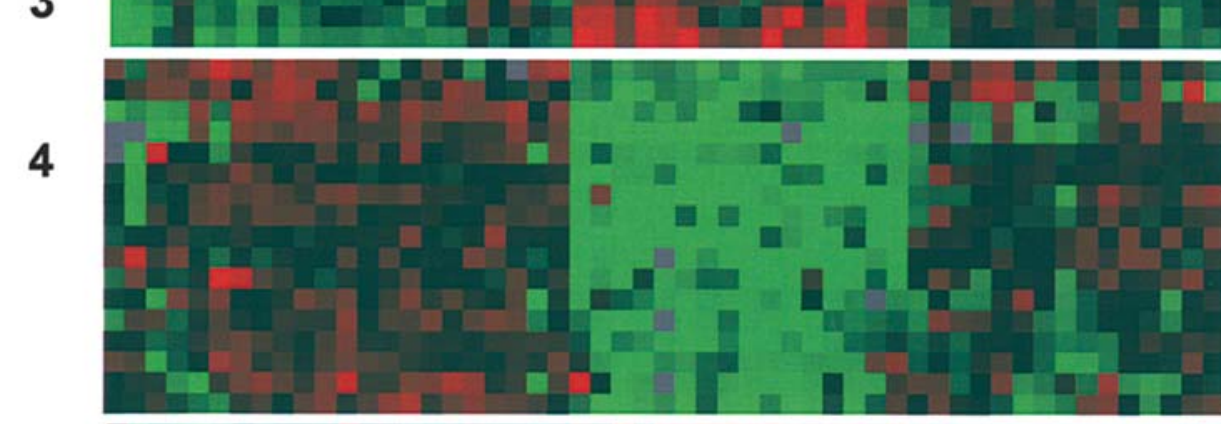

GRP58
GRP58

GRP58

RP58

NXF1 ASPSCA1 FLD22175 SLC9A1 PMM1 CESN? SESN2 TNFFSF1A MMP24 NTE NCSTN MOB-LAK GNLY

5

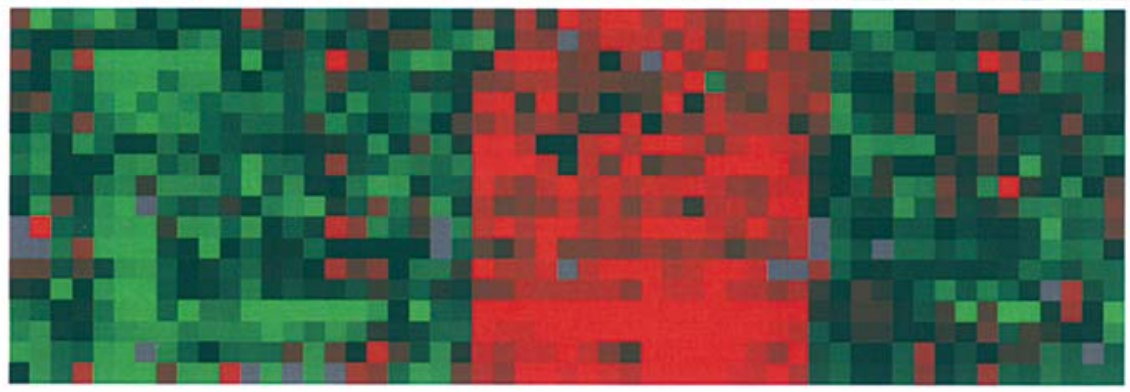

RNPC2

MGEAS
SMARCA5

BACH1

KPNB1

HNRPA2B1

YYZ

2AFZ

MGC33602

DAAP

DAB2

SEC22L3

PHC1

ATIC

ABHDE

FVT1

Figure 1. Clustering analysis of metastatic lung ADC and primary NSCLC. (A) Dendrogram of two-dimensional hierarchical clustering analysis using expression information of 4,423 genes for 53 samples consisting of 37 primary NSCLCs and 16 metastatic brain tumors from lung ADC. The color of each well represented by red and green indicates transcript levels above and below the median level of expression for each gene across all samples. Black color indicates unchanged expression and gray color indicates that expression was not detectable. In the horizontal axis, the 53 cases were clearly separated in three branches. In the vertical axis, the 4,423 genes were clustered in different branches according to their similarities in relative expression ratio. (B) Magnified views of representative parts of (A) demonstrating subclusters including gene clusters 4 and 5 (black bars in A). Gene cluster-1 of HIST2H2BE, cluster-2 of $H M G C L$, and cluster-3 of GRP58 indicate the three representative genes that were spotted on different positions on the slide glasses, demonstrating the reproducibility and reliability of our experiments. Gene clusters 4 and 5 indicated genes that were down-regulated and up-regulated in metastatic brain tumors, compared with other two histological types of tumor groups. 
A

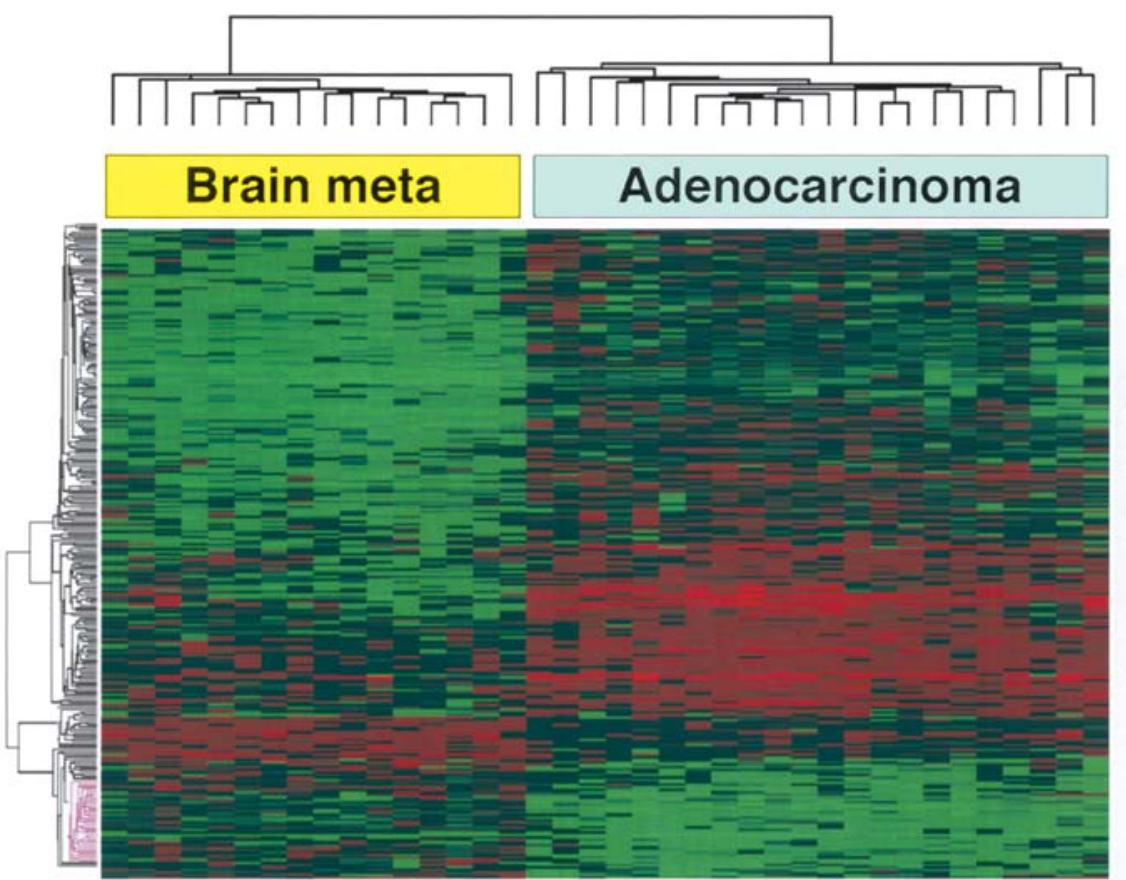

\section{4genes}

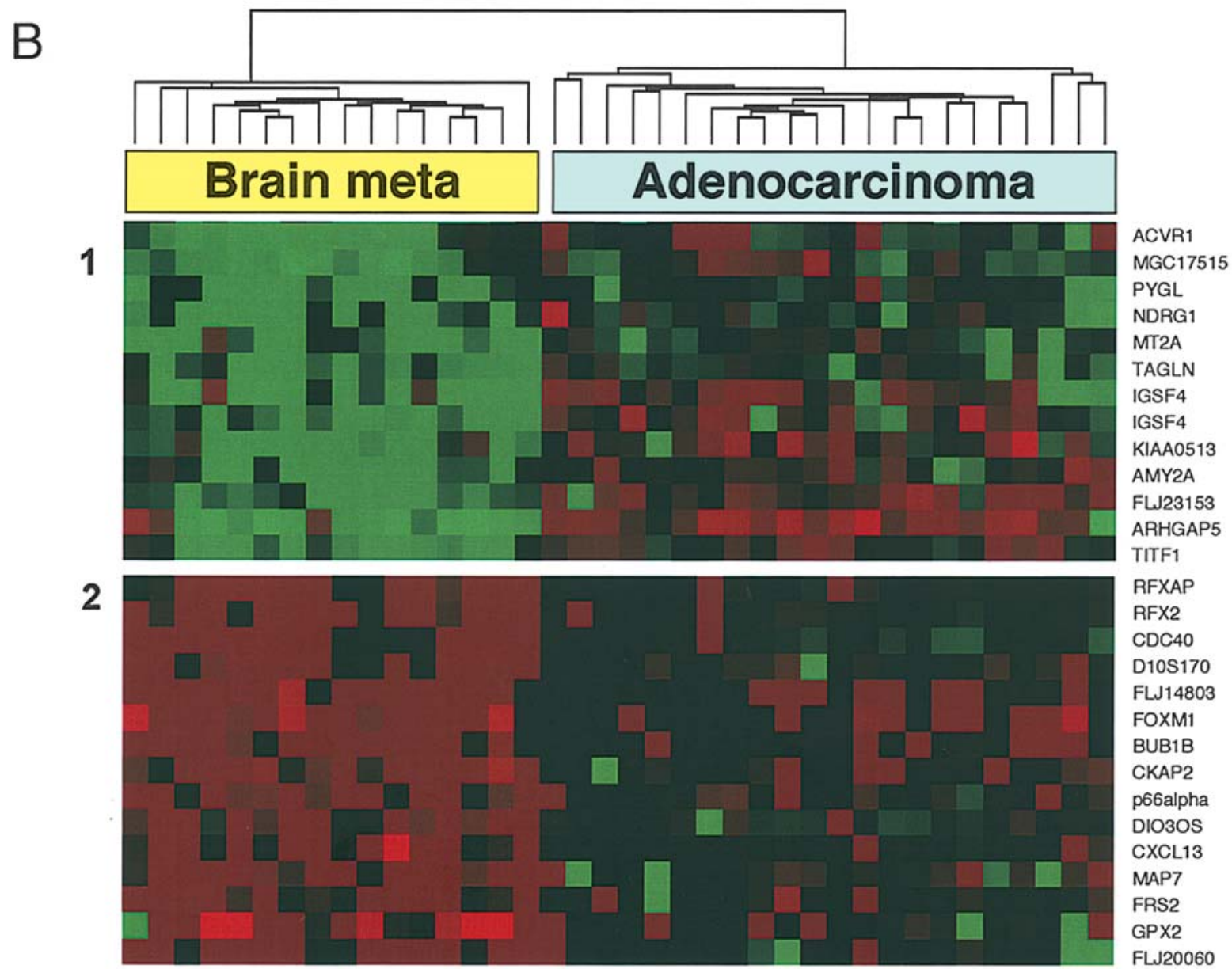

Figure 2. Genes showing differential expression between primary lung ADC and brain metastasis of ADC of the lung. (A) Two-dimensional hierarchical clustering analysis of 38 samples consisting of 22 primary lung ADCs and 16 metastatic brain tumors from lung ADC using 244 genes selected by a randompermutation test. In the horizontal axis, the 38 samples were classified into two trunks. In the vertical axis, 244 genes were clustered in different branches according to similarities in relative expression ratios. Genes in the lower main branch represent genes that were commonly up-regulated in metastatic brain tumors; those in the upper branch represent ones that revealed lower levels of expression in metastatic brain tumors than in primary ADCs. (B) Magnified views of representative parts of (A) showing genes relatively decreased (gene cluster-1), and increased (gene cluster-2) in brain metastatic legions compared with primary ADCs. 
previously reported to be down-regulated in various tumor types (22). TNFRSF1A (TNFRI) is widely known to induce apoptosis through activation of caspase-8 (23). C14ORF1, that is recently identified as a gene from the genome sequences and is conserved from yeast to mammalians (24), is considered to encode a membrane protein expressed abundantly in testis as well as various tumors. MMP24 (MT5-MMP) belonging to the membrane-bound matrix metalloproteinase family, was reported to be overexpressed in some histological types of brain tumors, but down-regulated in prostate cancers (25). The genes in cluster-5 that were overexpressed in metastatic tumor cells contained MGEA5 that was known to be overexpressed in meningioma and also detected in the patients' sera (26).

Genes showing differential expression between primary lung $A D C$ and brain metastatic foci of lung $A D C$. We compared the expression profiles of 22 primary lung ADCs with those of 16 metastatic lung ADCs to brain using a permutation test to select genes that might be applicable for separation of these two groups. As a result 244 genes were listed as candidates that showed the permutational P-value of $<0.001$. As shown in Fig. 2A, clustering algorithm using the 244 genes clearly separated primary and metastatic lung ADCs. Similar to that in Fig. 1B, identical genes of IGSF4, spotted on different positions on the slide glasses were also placed into the adjacent rows as shown in Fig. 2B, gene cluster-1. The top-ranked 20 genes that revealed the most significant differences in higher or lower expression between the two groups are listed in Table I. The genes included MT2A which was a candidate gene associated with liver metastasis of colon cancer in our previous study (Fig. 2B) $(27,28)$. RFX2 known as one of HLA II regulatory factors which drives HLA II genes expression via cis-acting DNA motif was expressed preferably in metastatic tumor cells (Fig. 2B, gene cluster-2). $R F X A P$, also known as HLA II regulatory factor by combining with RFXANK and RFX5, was also up-regulated in brain tumors (Fig. 2B, gene cluster-2). CXCL13, a ligand to chemokine receptors for CCR1, CCR3 and CXCR5, of which ligandreceptor pairs were recently indicated to play a critical role for distant metastasis, was also activated in brain metastasis (Fig. 2B, gene cluster-2) (29).

\section{Discussion}

Although recent development of chemotherapy and radiotherapy is so rapid and remarkable, most of cancer patients who have distant metastasis are incurable $(11,12)$. A large body of knowledge on genetic and epigenetic alterations occurring in cancers has been accumulated, but we are still unsuccessful in characterizing differences in biological nature of tumors in individual patients such as metastatic ability and the responses to the various treatments. Bernards and Weinberg have suggested that genes associated with metastasis might be already altered even in tumor cells at an early stage (30). We also observed through clinical evidence that certain types of tumor cells are highly metastatic compared to others. In fact, some patients are diagnosed to have lung cancer through the neurological symptoms caused by metastatic tumors to the brain as in the cases in this study.
On the other hand, patients diagnosed to have locally advanced NSCLCs are threatened by concurrent risk of local, regional and distant metastasis of the disease. Prophylactic cranial irradiation (PCI) is being investigated as treatment to prevent further brain metastasis and improve survival. Until now several studies have shown that PCI decreases the frequency of additional brain metastases in patients with either SCLC or NSCLC and improved survival $(11,31,32)$. Therefore, if we are able to predict a probability of brain metastases, we would be able to predict, for example, the patients who would have a benefit from PCI.

In this study, we selected cases with brain metastasis at a relatively earlier $\mathrm{pT}$ and/or $\mathrm{pN}$ stages of lung cancer. Generally, systemic spread of tumors cells detected as metastatic foci occurs when cancer has progressed and the size of primary tumors becomes relatively large in host organs. Hence, we considered that the brain metastatic cells examined in this study are likely to have a higher metastatic ability and also have a higher affinity for growth in the brain, and that a comparison of the expression profiles of metastatic lung ADC to brain examined in this study with those of surgically-resected primary ADC obtained in our previous study could help to find important key molecules associated with brain metastasis. The distinct gene-expression profiles we observed between primary lung ADCs, primary SCCs and brain metastatic sites from lung ADC (Fig. 1A) clearly suggested that primary ADCs without metastasis and metastatic ADCs have different biological nature. Because we analyzed three histopathological groups of lung tumors, two of which were histologically of the same origin, one can imagine that selection of genes could separate the three tumor types into two groups, one including lung SCC and the other containing both primary and metastatic ADCs of the lung. However, the computational analysis separated very clearly the group of metastatic tumors from primary ADCs. Since we were unable to obtain the primary lung tumor samples corresponding to brain tumors, it is unclear whether the primary tumors of these patients have different expression profiles from their metastatic tumors. However, our results suggest that these 16 metastatic tumors might have similar biological nature represented by similar gene expression profiles. The genes whose expression levels of metastatic tumors are commonly altered comparing to those of primary tumors could be associated with their highly metastatic ability, and may be applied to prediction of higher risk of metastasis and for development of molecular therapy.

When we compared 244 genes that revealed statistically different expression levels between primary lung carcinomas and distant metastatic legions, with those aberrantly expressed in liver metastasis of colon cancer $(27,28), D A T 1$, also known as $S L C 6 A 3$, and $M T 2 A$ were commonly down-regulated in the two different metastatic sites originated from colon and lung cancers. MT2A encodes metallothionein and is known to be induced by DNA damage and oxidative stress (33). In nasopharyngeal cancer, MT2A was overexpressed and associated with tumor proliferative activity (34).

We previously analyzed gene-expression profiles of 25 metastatic lesions from four organs (lung, liver, kidney and bone) using a metastasis model in mice with multiple organ dissemination by intravenous injection of human small cell 
Table I. Genes differently expressed between brain metastasis site and primary adenocarcinoma.

\begin{tabular}{|c|c|c|}
\hline Symbol & Description & P-value \\
\hline \multicolumn{3}{|c|}{ Up-regulated in metastatic site } \\
\hline CDC40 & Cell division cycle 40 homolog (yeast) & $1.07 \mathrm{E}-08$ \\
\hline FSCN3 & Fascin homolog 3, actin-bundling protein, testicular & 2.58E-07 \\
\hline$T A F 5 L$ & TAF5-like RNA polymerase II & 4.59E-07 \\
\hline ABHD6 & Abhydrolase domain containing 6 & $5.75 \mathrm{E}-07$ \\
\hline$R F X 2$ & Regulatory factor X, 2 (influences HLA class II expression) & 7.67E-07 \\
\hline FVT1 & Follicular lymphoma variant translocation 1 & $1.17 \mathrm{E}-06$ \\
\hline D10S170 & DNA segment on chromosome 10 (unique) 170 & $1.70 \mathrm{E}-06$ \\
\hline STAU & Staufen, RNA binding protein (Drosophila) & 4.42E-06 \\
\hline SH3GL2 & SH3-domain GRB2-like 2 & $5.52 \mathrm{E}-06$ \\
\hline RME8 & RME8 protein & $5.81 \mathrm{E}-06$ \\
\hline$H 2 A F Z$ & $\mathrm{H} 2 \mathrm{~A}$ histone family, member $\mathrm{Z}$ & $5.82 \mathrm{E}-06$ \\
\hline$S R P K 2$ & SFRS protein kinase 2 & $6.41 \mathrm{E}-06$ \\
\hline \multirow[t]{2}{*}{ LOC90557 } & Hypothetical protein BC016861 & $6.91 \mathrm{E}-06$ \\
\hline & EST & 7.33E-06 \\
\hline EEAl & Early endosome antigen $1,162 \mathrm{kDa}$ & 8.07E-06 \\
\hline MAP7 & Microtubule-associated protein 7 & $8.41 \mathrm{E}-06$ \\
\hline$A T P 1 B 3$ & ATPase, $\mathrm{Na}^{+} / \mathrm{K}^{+}$transporting, 33 polypeptide & 8.61E-06 \\
\hline CXCL13 & Chemokine (C-X-C motif) ligand 13 (B-cell chemoattractant) & $9.60 \mathrm{E}-06$ \\
\hline FLJ36031 & Hypothetical protein FLJ36031 & $1.07 \mathrm{E}-05$ \\
\hline TRAM2 & Translocation associated membrane protein 2 & $1.10 \mathrm{E}-05$ \\
\hline \multicolumn{3}{|c|}{ Down-regulated in metastatic site } \\
\hline$M G C 20446$ & Hypothetical protein MGC20446 & $8.68 \mathrm{E}-41$ \\
\hline$H L A-D P A 1$ & Major histocompatibility complex, class II, DP $\alpha 1$ & $3.58 \mathrm{E}-37$ \\
\hline FOLRl & Folate receptor 1 (adult) & $2.81 \mathrm{E}-30$ \\
\hline C9orf19 & Chromosome 9 open reading frame 19 & $8.64 \mathrm{E}-29$ \\
\hline CX3CLI & Chemokine (C-X3-C motif) ligand 1 & $1.21 \mathrm{E}-25$ \\
\hline NPC2 & Niemann-Pick disease, type C2 & $1.35 \mathrm{E}-23$ \\
\hline KIF5A & Kinesin family member $5 \mathrm{~A}$ & $3.32 \mathrm{E}-23$ \\
\hline WFDC2 & WAP four-disulfide core domain 2 & $3.56 \mathrm{E}-23$ \\
\hline$H A D H B$ & Hydroxyacyl-coenzyme A dehydrogenase & $6.30 \mathrm{E}-23$ \\
\hline$U B A P 1$ & Ubiquitin associated protein 1 & $1.53 \mathrm{E}-20$ \\
\hline $\mathrm{CTSH}$ & Cathepsin $\mathrm{H}$ & $2.26 \mathrm{E}-20$ \\
\hline DUSP6 & Dual specificity phosphatase 6 & $4.23 \mathrm{E}-20$ \\
\hline UNQ473 & $\mathrm{DMC}$ & 4.75E-20 \\
\hline$D A T 1$ & Neuronal specific transcription factor DAT1 & $7.12 \mathrm{E}-20$ \\
\hline FLJ32332 & Hypothetical protein FLJ32332 & 2.61E-19 \\
\hline$M U C 1$ & Mucin 1 , transmembrane & $3.56 \mathrm{E}-19$ \\
\hline PIAS3 & Protein inhibitor of activated STAT3 & $8.22 \mathrm{E}-19$ \\
\hline$N T E$ & Neuropathy target esterase & $9.00 \mathrm{E}-18$ \\
\hline$M G C 4309$ & Hypothetical protein MGC4309 & $1.21 \mathrm{E}-17$ \\
\hline PRSS8 & Protease, serine, 8 (prostasin) & 2.17E-17 \\
\hline
\end{tabular}

lung cancer (SCLC) cells (SBC-5). In a previous study, we extracted 435 genes that seemed to reflect the organ specificity of the metastatic cells (18). A comparison of the mouse model data and the results in this study identified 21 genes including CXCL13, SPTBN1, and MARCKS that were commonly deregulated in various metastatic target organs; $M A R C K S$ alters its subcellular localization from plasma membrane to cytoplasm depending on its phosphorylation status and influence to the cell shape (35). Some extracellular matrix proteins such as COLIA1, ACTA1, and cellular antigens belonging to the Eph and integrin families were reported to be key molecules playing critical roles for malignant phenotype through cDNA microarray analysis of various kinds of cell lines and primary melanomas (36). RhoC-related proteins, ARHGDIB and ARHGAP5, were also suggested to be associated with metastatic feature of melanomas (6). 
Alteration of expression levels of these genes might affect actomyosin-based cytoskeletal filaments and cell-cell interaction, and enhance cell motility.

In conclusion, we identified dozens of molecules that might be associated with brain metastasis of lung cancer and would provide useful information to comprehensive understanding of lung carcinogenesis. To our knowledge, this is the first genome-wide gene expression analysis of brain metastasis of clinical NSCLCs. The genes or gene products identified might serve as novel diagnostic and predictive markers for disease progression, and for development of new therapeutic approaches that could lead to molecular targetbased chemotherapy and prevention of metastasis.

\section{Acknowledgements}

This work was supported in part by the 'Research for the Future' Program Grant of The Japan Society for the Promotion of Science (\#00L01402) to Y.N.

\section{References}

1. Chambers AF, Groom AC and MacDonald IC: Dissemination and growth of cancer cells in metastatic sites. Nat Rev Cancer 2: 563-572, 2002.

2. Al-Mehdi AB, Tozawa K, Fisher AB, Shientag L, Lee A and Muschel RJ: Intravascular origin of metastasis from the proliferation of endothelium-attached tumor cells: a new model for metastasis. Nat Med 6: 100-102, 2000.

3. Fidler IJ, Yano S, Zhang RD, Fujimaki T and Bucana CD: The seed and soil hypothesis: vascularisation and brain metastases. Lancet Oncol 3: 53-57, 2002.

4. Paget $\mathrm{S}$ : The distribution of secondary growths in cancer of the breast. Cancer Metastasis Rev 8: 98-101, 1989.

5. Muller A, Homey B, Soto $\mathrm{H}$, et al: Involvement of chemokine receptors in breast cancer metastasis. Nature 410: 50-56, 2001.

6. Clark EA, Golub TR, Lander ES and Hynes RO: Genomic analysis of metastasis reveals an essential role for RhoC. Nature 406: 532-535, 2000.

7. Lauffenburger DA and Horwitz AF: Cell migration: a physically integrated molecular process. Cell 84: 359-369, 1996.

8. Kikuchi T, Daigo Y, Katagiri T, et al: Expression profiles of non-small cell lung cancers on cDNA microarrays: identification of genes for prediction of lymph-node metastasis and sensitivity to anti-cancer drugs. Oncogene 22: 2192-2205, 2003.

9. Hoffman PC, Mauer AM and Vokes EE: Lung cancer. Lancet 355: 479-485, 2000.

10. Robnett TJ, Machtay M, Stevenson JP, Algazy KM and Hahn SM: Factors affecting the risk of brain metastases after definitive chemoradiation for locally advanced non-small cell lung carcinoma. J Clin Oncol 19: 1344-1349, 2001.

11. Pottgen $\mathrm{C}$ and Stuschke $\mathrm{M}$ : The role of prophylactic cranial irradiation in the treatment of lung cancer. Lung Cancer 33: S153-S158, 2001.

12. Hall WA, Djalilian HR, Nussbaum ES and Cho KH: Long-term survival with metastatic cancer to the brain. Med Oncol 17: 279-286, 2000

13. Beer DG, Kardia SL, Huang CC, et al: Gene-expression profiles predict survival of patients with lung adenocarcinoma. Nat Med 8: 816-824, 2002.

14. Arnold SM, Young AB, Munn RK, Patchell RA, Nanayakkara N and Markesbery WR: Expression of p53, bcl-2, E-cadherin, matrix metalloproteinase-9, and tissue inhibitor of metalloproteinases-1 in paired primary tumors and brain metastasis. Clin Cancer Res 5: 4028-4033, 1999.

15. D'Amico TA, Aloia TA, Moore MB, Conlon DH, Herndon JE II, Kinch MS and Harpole DH Jr: Predicting the sites of metastases from lung cancer using molecular biologic markers. Ann Thorac Surg 72: 1144-1148, 2001.
16. Milas I, Komaki R, Hachiya T, et al: Epidermal growth factor receptor, cyclooxygenase-2, and $\mathrm{BAX}$ expression in the primary non-small cell lung cancer and brain metastases. Clin Cancer Res 9: 1070-1076, 2003.

17. Bubb RS, Komaki R, Hachiya T, et al: Association of Ki-67, p53, and bcl-2 expression of the primary non-small cell lung cancer lesion with brain metastatic lesion. Int J Radiat Oncol Biol Phys 53: 1216-1224, 2002.

18. Kakiuchi S, Daigo Y, Tsunoda T, Yano S, Sone S and Nakamura Y: Genome-wide analysis of organ-preferential metastasis of human small cell lung cancer in mice. Mol Cancer Res 1: 485-499, 2003.

19. Kakiuchi S, Daigo Y, Ishikawa N, et al: Prediction of sensitivity of advanced non-small cell lung cancers to gefitinib (Iressa, ZD1839). Hum Mol Genet 13: 3029-3043, 2004.

20. Ochi K, Daigo Y, Katagiri T, et al: Expression profiles of two types of human knee-joint cartilage. J Hum Genet 48: 177-182, 2003.

21. Ochi K, Daigo Y, Katagiri T, et al: Prediction of response to neoadjuvant chemotherapy for osteosarcoma by gene-expression profiles. Int J Oncol 24: 647-655, 2004

22. Spieker N, Beitsma M, van Sluis P, et al: An integrated 5-Mb physical, genetic, and radiation hybrid map of a 1 p36.1 region implicated in neuroblastoma pathogenesis. Genes Chromosomes Cancer 27: 143-152, 2000.

23. Ahonen M, Poukkula M, Baker AH, Kashiwagi M, Nagase H, Eriksson JE and Kahari VM: Tissue inhibitor of metalloproteinases-3 induces apoptosis in melanoma cells by stabilization of death receptors. Oncogene 22: 2121-2134, 2003.

24. Veitia RA, Ottolenghi C, Bissery MC and Fellous A: A novel human gene, encoding a potential membrane protein conserved from yeast to man, is strongly expressed in testis and cancer cell lines. Cytogenet Cell Genet 85: 217-220, 1999.

25. Llano E, Pendas AM, Freije JP, Nakano A, Knauper V, Murphy G and Lopez-Otin C: Identification and characterization of human MT5-MMP, a new membrane-bound activator of progelatinase a overexpressed in brain tumors. Cancer Res 59: 2570-2576, 1999.

26. Heckel D, Comtesse N, Brass N, Blin N, Zang KD and Meese E: Novel immunogenic antigen homologous to hyaluronidase in meningioma. Hum Mol Genet 7: 1859-1872, 1998.

27. Li M, Lin YM, Hasegawa S, et al: Genes associated with liver metastasis of colon cancer, identified by genome-wide cDNA microarray. Int J Oncol 24: 305-312, 2004.

28. Yanagawa R, Furukawa Y, Tsunoda T, et al: Genome-wide screening of genes showing altered expression in liver metastases of human colorectal cancers by cDNA microarray. Neoplasia 3: 395-401, 2001.

29. Houshmand P and Zlotnik A: Therapeutic applications in the chemokine superfamily. Curr Opin Chem Biol 7: 457-460, 2003.

30. Bernards R and Weinberg RA: A progression puzzle. Nature 418: 823, 2002.

31. Auperin A, Arriagada R, Pignon JP, et al: Prophylactic cranial irradiation for patients with small cell lung cancer in complete remission. Prophylactic Cranial Irradiation Overview Collaborative Group. N Engl J Med 341: 476-484, 1999.

32. Stuschke M, Eberhardt W, Pottgen C, et al: Prophylactic cranial irradiation in locally advanced non-small cell lung cancer after multimodality treatment: long-term follow-up and investigations of late neuropsychologic effects. J Clin Oncol 17: 2700-2709, 1999

33. Perez MJ and Cederbaum AI: Metallothionein 2A induction by zinc protects HEPG2 cells against CYP2E1-dependent toxicity. Free Radic Biol Med 34: 443-435, 2003.

34. Tan OJ, Bay BH and Chow VT: Differential expression of metallothionein isoforms in nasopharyngeal cancer and inhibition of cell growth by antisense down-regulation of metallothionein2A. Oncol Rep 13: 127-131, 2005.

35. Disatnik MH, Boutet SC, Pacio W, Chan AY, Ross LB, Lee CH and Rando TA: The bi-directional translocation of MARCKS between membrane and cytosol regulates integrin-mediated muscle cell spreading. J Cell Sci 117: 4469-4479, 2004.

36. Bittner M, Meltzer P, Chen Y, et al: Molecular classification of cutaneous malignant melanoma by gene expression profiling. Nature 406: 536-540, 2000. 